\title{
DESIGN AND PERFORMANCE ANALYSIS OF GRAVITY ASSISTED POWER (GAP) GENERATING SYSTEM FOR HARVESTING ELECTRICAL ENERGY
}

\author{
Dr.J.Arputha Vijaya Selvi ${ }^{1}$ and S. Devayani ${ }^{2}$
}

\begin{abstract}
In this paper, a system for harvesting electrical energy through Gravity Assisted Power (GAP) using perpetual motion is presented. The objective is to build a device which gives energy for $24 \times 7$ hours without external energy and pileup. The proposed system consists of a weighted pendulum bounded by a copper wire oscillates by perpetual motion between two magnets arranged in a parallel manner. When the pendulum oscillates it cuts the magnetic field and hence emf is produced. The harvested energy is transmitted through wireless power transmission (WPT) system. Also DC power generated can be stored via batteries and used by the local population to use for lamps and other basic utilities that may be take for granted on a daily basis.
\end{abstract}

Keywords - Electrical energy, Perpetual motion, Gravity Assisted Power.

\section{INTRODUCTION}

All around the world people are trying to generate new forms of energy to satisfy their increasing needs. Generally energy harvesting using natural resources like airflow, sunlight etc., is entirely different from energy generation involving the of non-renewable energy such as fossil fuels, lignite etc. The main difference is that for energy generation, the resources must be available in the direct environment for the application. First-generation technologies include fossil fuels, lignite etc. A fossil fuel power station has machinery to convert the heat energy into mechanical energy, which then operates an electrical generator. Variations between countries generating electrical power are observed where only $10 \%$ of electricity is generated from non-transient source like fossil fuels in France, US and China are at higher rate of production with $70 \%$ and $80 \%$ respectively. In US, other than any source, power generation causes around $40 \%$ of the hazardous emissions. Also, fossil fuel combustion for electricity generation is the major reason for $65 \%$ of the emissions of $\mathrm{SiO} 2$, which is the main component causing acid rain. Another existing energy generation systems includes nuclear power generation which costs high. Second-generation technologies include solar, wind, hydro and other power generation systems. In 2015, Hydropower generated was $16.6 \%$ of the world total electricity and $70 \%$ of all renewable electricity. It also disturbs the water flow and lower the dissolved oxygen levels in water which will affect the underwater species. Hydropower may be affected by drought. Airflows can be used to run wind turbines. Wind power must compete with conventional generation sources on a construction cost basis. Turbine blades could damage local wildlife. The proposed system overcomes some of these drawbacks, with a new method of power generation using the Gravitational force which is naturally present $24 * 7$ hours. As because gravity is present everywhere, it can be easily utilized by anyone on the Earth. It will be an added advantage because Energy crisis is increasing at a faster pace and hence within a few years, there will be shortage of fuels for power generation and the other renewable energy like solar, wind, biomass, etc., are only available during the day/night. This present invention relates to a device for conversion of gravitational energy to electrical energy which can be made available at a low cost to the common man. "GRAVITY ASSISTED POWER GENERATION SYSTEM" (GAP) system is the emerging technology in the power generation field which overcomes all drawbacks in the existing systems. In the proposed system, perpetual motion generation to oscillate

\footnotetext{
${ }^{1}$ Department of Electronics and Communication Engineering Kings College of Engineering, Thanjavur, Tamilnadu, India

${ }^{2}$ Department of Electronics and Communication Engineering Kings College of Engineering, Thanjavur, Tamilnadu, India
} 


\section{BACKGROUND AND RELATED WORKS}

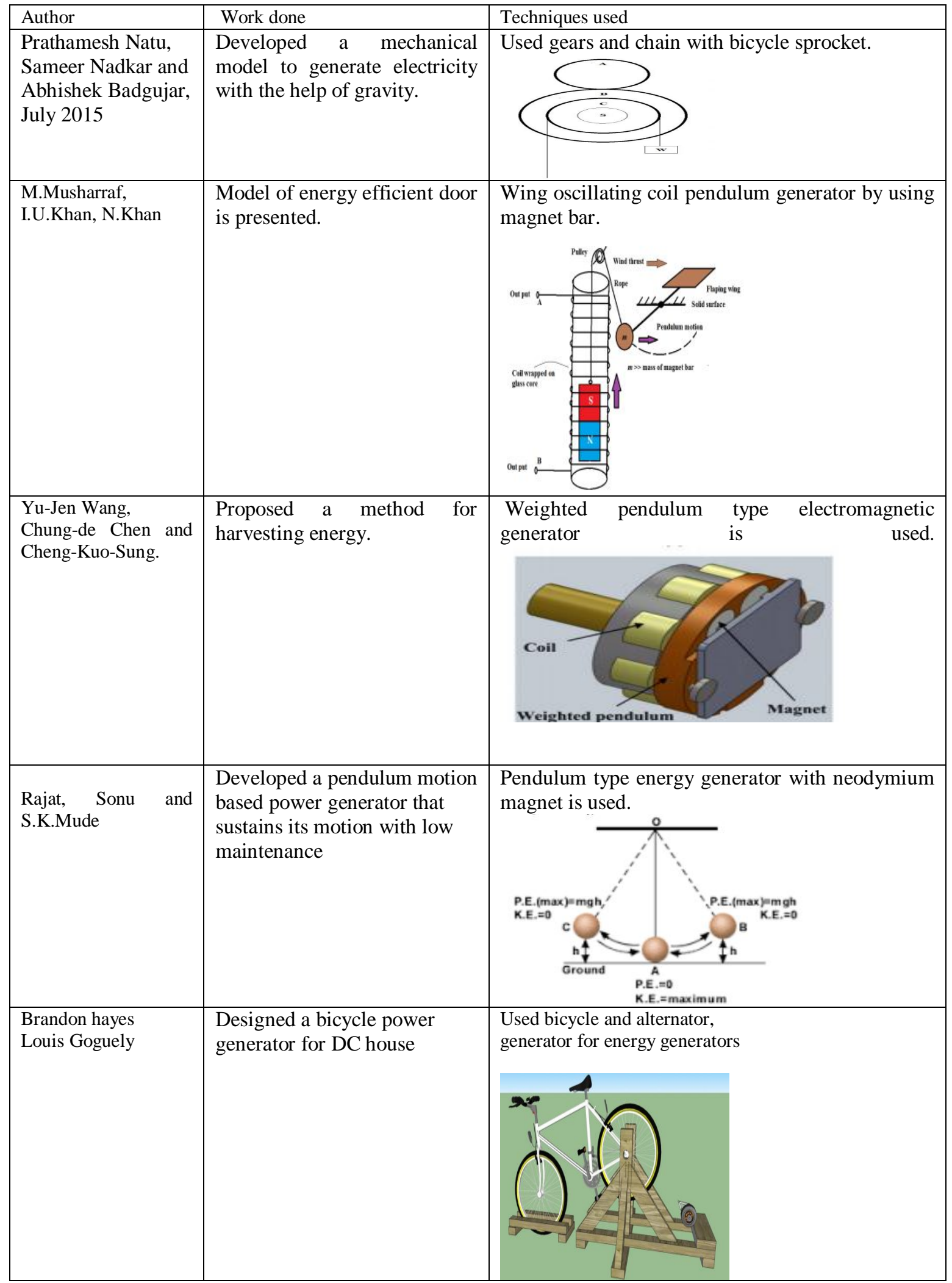




\section{EXPERIMENTAL SETUP}

The proposed work consists of three important modules as shown in Fig. 1. They are (i) GAP system with perpetual motion generator construction (ii) Performance analysis and validation system (iii) WPT System.

\subsection{GAP system with perpetual motion generator construction:}

The setup is basically meant for lighting up a commercial LED lamp by converting the mechanical energy of the pendulum oscillation into electrical energy. To develop green technology model for generating electricity, Gravity assisted power generating system (GAP) based on the concept of Gravitational force is used. The experimental setup, based on the concept of simple pendulum, consists of magnets and copper coil assembly where coil and its associated components are wound over the pendulum. At the two sides of the pendulum, magnet bar is fixed and magnetic flux is produced around the magnets. Whenever coil is moved back and forth it cuts the magnetic flux produced by the magnets and an emf induced. Gravity Assisted Power (GAP) generator system doesn't require any sources of energy. It's an autonomous/ standalone energy system. On the contrary, this setup consists of perpetual motion assembly comprises a CPU fan with magnets been placed in the blades of the fan by which magnetic poles exert forces on each other in such a way that like poles repel and unlike poles attract each other which makes the assembly rotates continuously.

Then a mechanical setup is employed, which resembles a pulley, with its one side gets connected to the CPU fan assembly and on the other side to the simple pendulum. On using this entire assembly the simple pendulum coil that suspends between strong magnets endlessly. In conventional methods, an initial force is applied manually to the gravity assisted rotational mechanism to make it rotate continuously. To validate and analyse the developed model for different dimensions/values of components to get a maximum energy, modelling techniques and simulation process are used.

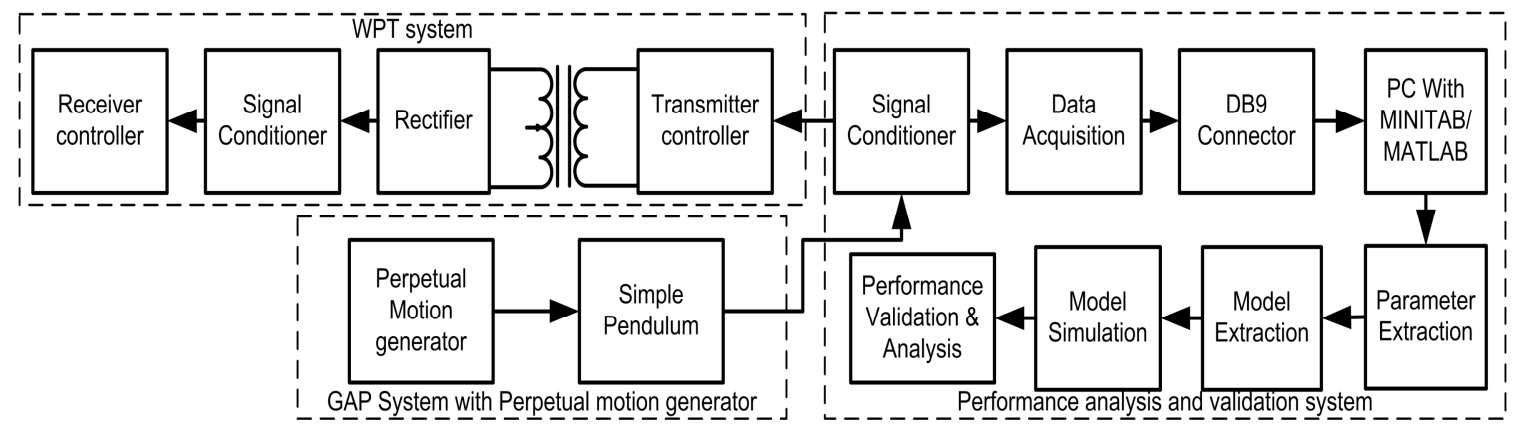

Figure 1. Experimental setup of GAP system

\subsection{Basic setup /components of pendulum:}

A simple pendulum consists of a weighted object which is hung by a string with a fixed support. That object here denotes the pendulum bob. When the bob is disturbed from its equilibrium position, it begins to oscillate back and forth vibration between the magnets. The amplitude $\theta^{\circ}$ of a pendulum is given as the maximum angular deviation from the equilibrium position of the object. Frequency $f$, is given by number of oscillations from one side to the other made by a pendulum per second. The time taken $\mathrm{T}$ for one oscillation of a pendulum is dependent only upon the length $\mathrm{L}$ of the pendulum and also the weight of the object. Perpetual Motion generator is used to generate a energy that oscillates the simple pendulum with infinite period or till the external force applied to stop the oscillation. Perpetual motion is generated through the CPU fan like assembly in which the magnets are placed on each wing and common magnet with respect to the all wings is placed on outer chase of the CPU fan. Due to the attraction reflection of the magnets, fan rotates continuously thus energy is generated. Generated energy is coupled on suspension point of the simple pendulum through pulley. 


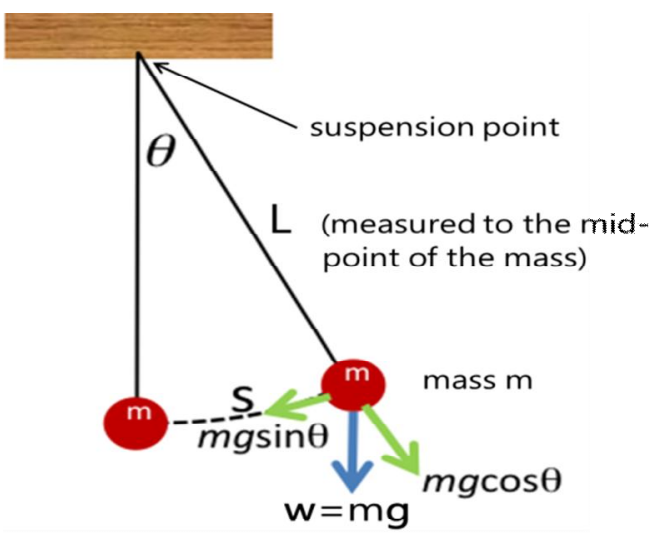

Figure 2. Simple pendulum

\subsection{Performance analysis and validation of system}

The output of the setup is sent to the signal digitizer. Using digitizer, analog signals that are continuously varying in the given period of time are converted into digital signals that are discrete. That digitized data is in the form of binary numbers, which facilitate computer processing and other operations, is given to two modules (Data acquisition unit and WPT block). Data acquisition control unit logs the data and it is plotted in the Minitab. The curve is fitted for the plot and the parameters are extracted. As per the Faraday's law an emf is produced when a coil is dropped in the magnetic field. When the pendulum is made to oscillate it cuts the magnetic field and thus emf is produced. The model is tested for various dimensions of magnets/ various types of magnets, various number of coil turns to get the sufficient energy. The entire setup can be modeled using modeling tools such as MINITAB.

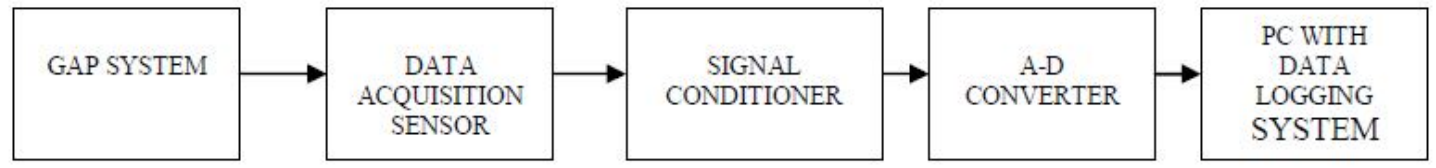

Figure 3. Block schematic of data acquisition system

\subsection{System validation}

The simulation is done in both Minitab and Matlab. The equations are obtained for both and it is taken for the overall analysis. The experiments are repeated for two coils. In Minitab, Many factors/inputs/variables must be taken into consideration when making a product especially a brand new one Based on a Design of Experiments (DOE) methodology for determining parameter levels. The simulation and modelling is done in Minitab for better accuracy. And moreover Minitab provides a statistical analysis of a product performance. It shows the signal to noise ratio for the entire performance of the product. Minitab software shows the necessary result which is needed for the quality performance of the design. Logging of data is an important parameter from which we can get the values and that can be simulated and designed into a model.

\section{$2.5 \quad$ Wireless Power Transmission system}

WPT consists of a "transmitter" and "receiver'. The transmitter is connected to a simple pendulum setup which is called source of power, which converts the power to a time-varying electromagnetic field, and one or more "receiver" devices which receive the power and convert it back to DC or AC electric current which is used by an electrical load.

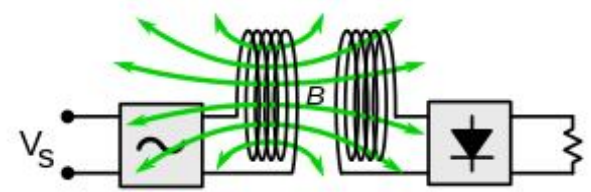

Figure 4. Schematic of Wireless Power Transmission system 
Table -1 Experiment Result

Table 1 Validation of systems performance when $\mathrm{N}=50$.

\begin{tabular}{|l|l|l|l|}
\hline $\mathrm{N}=50$ & $\mathrm{T0}(\mathbf{m m})$ & $\begin{array}{l}\text { Output Voltage } \\
(\mathrm{V})\end{array}$ \\
\hline S. No & L(m) & 1 & 3.4 \\
\hline 1. & 10 & 1.5 & 4.5 \\
\hline 02. & 20 & & \\
\hline
\end{tabular}

Table 2 Validation of systems performance when $\mathrm{L}=50 \mathrm{~m}$

\begin{tabular}{|l|l|l|l|}
\hline L=50m & N & T(mm) & $\begin{array}{l}\text { Output Voltage } \\
(\text { V) }\end{array}$ \\
\hline S. No & N & \\
\hline 1. & 500 & $.5 m m$ & 3.7 \\
\hline 2. & 1000 & $1 \mathrm{~mm}$ & 5.2 \\
\hline
\end{tabular}

Table 3 Validation of systems performance when $\mathrm{T}=0.5 \mathrm{~mm}$.

\begin{tabular}{|c|c|c|c|}
\hline \multicolumn{4}{|c|}{$\mathrm{T}=0.5 \mathrm{~mm}$} \\
\hline S. No & $\mathbf{N}$ & $\mathbf{L}(\mathbf{m})$ & $\begin{array}{ll}\text { Output } & \text { Voltage } \\
\text { (v) }\end{array}$ \\
\hline 1. & 500 & .5 & 3.9 \\
\hline 2. & 1000 & 1 & 5.6 \\
\hline
\end{tabular}

The equation for analysis and validation comprises three parameters such as Rest time (T), Angle of oscillation $(\theta)$, Number of oscillation $(\mathrm{N})$ by using curve fitting tool in MATLAB.

$$
f(x, y)=a+b^{*} \sin \left(m^{*} \text { pi }^{*} x^{*} y\right)+c^{*} \exp \left(-\left(w^{*} y\right)^{\wedge} 2\right)
$$

Where $\mathrm{X}=$ Angle of oscillaton, $\mathrm{Y}=$ No.of oscillation $\mathrm{Z}=$ Rest time $\mathrm{a}, \mathrm{b}$ and $\mathrm{c}$ are coefficients of $\mathrm{X}, \mathrm{Y}$ and $\mathrm{Z}$ for the given data set.

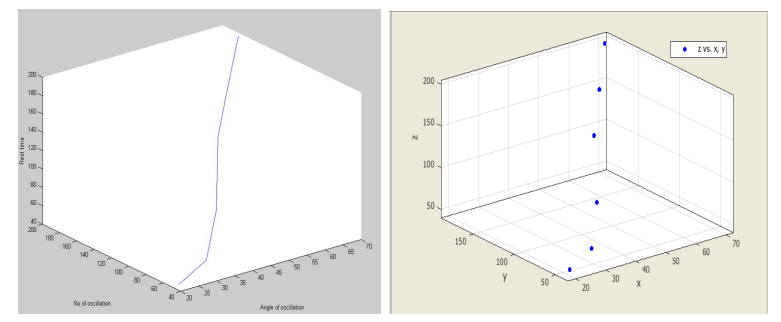

Figure 5. Simulation results- Relation shows between $\mathrm{T}, \theta, \mathrm{N}$

\section{IV.CONCLUSION}

In this paper model to convert gravity energy into electrical energy by oscillating simple pendulum using perpetual motion is proposed. The performance of the system is analyzed by varying the simple pendulum parameters such as number of turns, length of the pendulum and thickness of the coil. Among the three methods adopted, the system produces maximum efficiency when increasing the length of the pendulum.

\section{ACKNOWLEDGEMENT}

The authors express their sincere gratitude to the Management of Kings College of Engineering, Thanjavur, India, for being the source of inspiration for the successful completion of this work. Also the authors would like to sincerely thank Foundation for Advancement of Education and Research (FAER), Motorola Group, Bangalore, for the partial financial assistance towards the completion of this work.

\section{REFERENCES}

[1] Prathamesh Natu, Sameer Nadkar and Abhishek Badgujar, "generation of electricity using gravity" International Journal of Mechanical Engineering and Technology (IJMET) Volume 6, Issue 7, pp. 79-84, July 2015.

[2] Huang, Ting Yen "Gravity assisted rotational mechanism" European patent application no, EP 2359918 A2, Aug 2011.

[3] Gomez Nacer "Gravity generator of electricity" united states patent application Norwel, no 0012058, Jan 2007. 


\section{Design And Performance Analysis Of Gravity Assisted Power (Gap) Generating System For Harvesting Electrical Energy

[4] Rajat, Sonu and S.K.Mude, "Electrical Energy Generation By using Pendulum Motion”, International Journal of Advance Research , Ideas and Innovations in Technology, Volume2, Issue2, pp 1-5, 2016.

[5] A. Asim K Sen, "Generation of electricity using gravitational energy," united states patent application no. 4980578 , Dec 1990.

[6] Brandon Hayes Louis Goguely "Bicycle Power Generator Design for DC House: Off Grid Energy Solutions.

[7] Y. J. Wang, C. D. Chen and C. K. Sung, "System Design of a Weighted-Pendulum-Type Electromagnetic Generator for Harvesting Energy From a Rotating Wheel," in IEEE/ASME Transactions on Mechatronics, vol. 18, no. 2, pp. 754-763, April 2013.

[8] M.Musharraf, I.U.Khan, N.Khan "Design of an Oscillating Coil Pendulum Energy Generating System" the 4th International Conference on Sustainable Energy Information Technology (SEIT-2014).

[9] Brando Hayes, Louis Goguely”Bicycle Power Generation Design For DC House: Off Grid Energy Solution” 2011.

[10] Martin Philip Riddiford, "Gravity-Powered Electrical Energy Generators”, London (GB, Pub. No.: US 2012/0212948 A1 Pub. Date: Aug. $23,2012$.

[11] I.Tudor, F. Leach , C. Bobean, V. Pavel, and I. Vadan."Study and design of a wing oscillating wind system.”Advanced Topics in Electrical Engineering (ATEE),2011 7th International Symposium on IEEE, 2011: pp 1-4.

[12] N. Ida.”Design and control of a magnetic pendulum actuator. “Optimization of Electrical and Electronic Equipment (OPTIM), 2012 13 International Conference on. IEEE, (2012.) 